\title{
A systematic study of excited-state effects on nucleon axial form factors
}

\author{
G.M. von Hippel ${ }^{* 1}$, D. Djukanovic ${ }^{2}$, J. Hua ${ }^{1}$, B. Jäger ${ }^{3}$, P. Junnarkar ${ }^{2}$, H.B. Meyer ${ }^{1,2}$, \\ T.D. $\mathbf{R a e}^{4}$, H. Wittig ${ }^{1,2}$ \\ ${ }^{1}$ PRISMA Cluster of Excellence and Institute of Nuclear Physics, University of Mainz, \\ 55099 Mainz, Germany \\ ${ }^{2}$ Helmholtz-Institute Mainz, University of Mainz, 55099 Mainz, Germany \\ ${ }^{3}$ Department of Physics, College of Science, University of Swansea, Swansea SA2 8PP, U.K. \\ ${ }^{4}$ Bergische Universität Wuppertal, Gaußstraße 20, 42119 Wuppertal, Germany \\ E-mail: hippel@uni-mainz.de
}

\begin{abstract}
We extend our study of excited-state effects on nucleon vector form factors to the case of the axial vector and pseudoscalar form factors. Combining information from a variety of different ratios of two- and three-point functions, we are able to extract the form factors $G_{A}$ and $G_{P}$ over a range of momentum transfers $Q^{2}$; together with the use of different methods to suppress excited-state contaminations this allows us to systematically study the effect of excited states.
\end{abstract}

The 33rd International Symposium on Lattice Field Theory

14 -18 July 2015

Kobe International Conference Center, Kobe, Japan

${ }^{*}$ Speaker. 


\section{Introduction}

The axial and pseudoscalar form factors of nucleon defined by

$$
\left\langle N\left(p^{\prime}, s^{\prime}\right)\left|A_{\mu}(0)\right| N(p, s)\right\rangle=\bar{u}_{s^{\prime}}\left(p^{\prime}\right)\left(G_{A}\left(Q^{2}\right) \gamma_{\mu} \gamma_{5}+\frac{q_{\mu}}{2 m_{N}} G_{P}\left(Q^{2}\right) \gamma_{5}\right) u_{s}(p),
$$

where $q=p^{\prime}-p$ and $Q^{2}=-q^{2}$, are valuable and important predictions from lattice $\mathrm{QCD}$, provided all systematics are understood.

Experimentally, $G_{A}\left(Q^{2}\right)$ is accessible via pion electroproduction and elastic neutrino scattering; $G_{A}(0)=g_{A}$ is measured very precisely in neutron $\beta$ decay [1]. $G_{P}\left(Q^{2}\right)$ is experimentally measured in muon capture on the proton, and is only poorly known.

Previous studies of the axial charge of the nucleon [2] found that accounting for excited-state effects was crucial in reproducing the experimental value. Our study of nucleon electromagnetic form factors [3] found that a systematic treatment of excited-state contaminations was essential in order to reproduce the experimental values of the nucleon charge radii. This leads to the expectation that excited-state effects will likewise be important in studying the axial form factors of the nucleon.

\section{Methods}

\subsection{Lattice measurements}
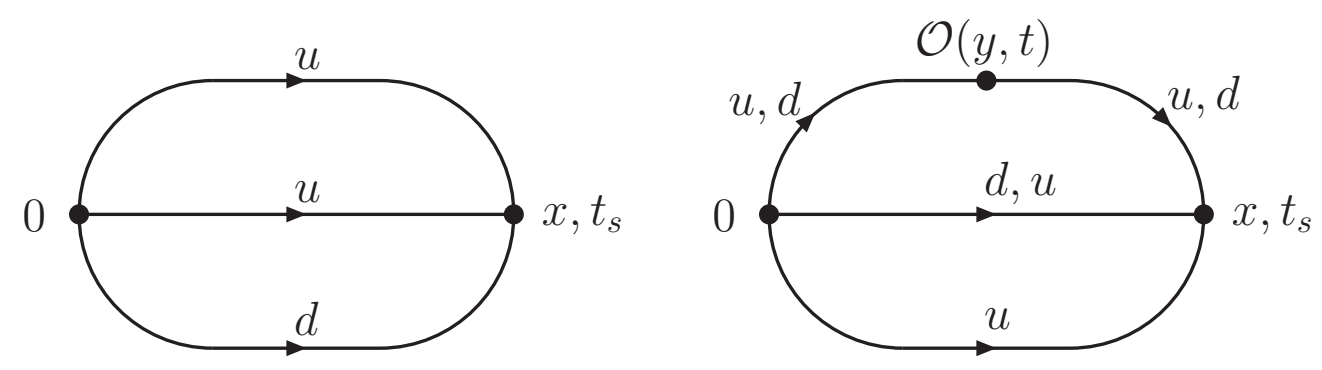

Figure 1: Quark-level diagrams for the nucleon two- and three-point functions.

On the CLS $N_{\mathrm{f}}=2$ ensembles, we measure the two- and three-point functions

$$
C_{2}\left((p, t)=\sum_{x} \mathrm{e}^{i p \cdot x} \Gamma_{\beta \alpha}\left\langle\Psi^{\alpha}(x, t) \bar{\Psi}^{\beta}(0)\right\rangle, \quad C_{3, \mathscr{O}}\left(q, t, t_{s}\right)=\sum_{x, y} \mathrm{e}^{i q \cdot y} \Gamma_{\beta \alpha}\left\langle\Psi^{\alpha}\left(x, t_{s}\right) \mathscr{O}(y, t) \bar{\Psi}^{\beta}(0)\right\rangle\right.
$$

for $\mathscr{O} \in\left\{A_{\mu}^{I}, P\right\}$, where we use the polarization matrix $\Gamma=\frac{1}{2}\left(1+\gamma_{0}\right)\left(1+i \gamma_{5} \gamma_{3}\right)$ and the improved current $A_{\mu}^{I}=A_{\mu}+a c_{A} \partial_{\mu} P$. To reduce excited-state contaminations from the outset, we use Gaussian smearing [4] with APE-smeared [5] links at source and sink; for the three-point functions, the extended-propagator method [6] is used.

\subsection{Ratios and decomposition}

Forming the ratios

$$
R_{\mathscr{O}}\left(q, t, t_{s}\right)=\frac{C_{3, \mathscr{O}}\left(q, t, t_{s}\right)}{C_{2}\left(q, t_{s}\right)} \sqrt{\frac{C_{2}\left(q, t_{s}-t\right) C_{2}(0, t) C_{2}\left(0, t_{s}\right)}{C_{2}\left(0, t_{s}-t\right) C_{2}(q, t) C_{2}\left(q, t_{s}\right)}}
$$


for $\mathscr{O} \in\left\{A_{\mu}^{I}, P\right\}$, the form factors can be extracted via their asymptotic behaviour $\left(t_{s} \gg t \gg 0\right)$,

$$
\begin{aligned}
& R_{A_{0}^{I}}\left(q, t, t_{s}\right) \rightarrow \frac{q_{3}}{\sqrt{2 E_{q}\left(m_{N}+E_{q}\right)}}\left(G_{A}\left(q^{2}\right)+\frac{m_{N}-E_{q}}{2 m_{N}} G_{P}\left(q^{2}\right)\right), \\
& R_{A_{k}^{I}}\left(q, t, t_{s}\right) \rightarrow \frac{i}{\sqrt{2 E_{q}\left(m_{N}+E_{q}\right)}}\left(\left(m_{N}+E_{q}\right) \delta_{k 3} G_{A}\left(q^{2}\right)-\frac{q_{3} q_{k}}{2 m_{N}} G_{P}\left(q^{2}\right)\right), \\
& R_{P}\left(q, t, t_{s}\right) \rightarrow \frac{q_{3}}{\sqrt{2 E_{q}\left(m_{N}+E_{q}\right)}}\left(\frac{m_{N}}{m_{P C A C}} G_{A}\left(q^{2}\right)+\frac{q^{2}}{4 m_{N} m_{P C A C}} G_{P}\left(q^{2}\right)\right) .
\end{aligned}
$$

This decomposition suggests two possible strategies to extract $G_{A}, G_{P}$ :

1. Strategy I:

(a) Extract asymptotic behaviour of $R_{\mathscr{O}}\left(q, t, t_{s}\right) \rightarrow R_{\mathscr{O}}^{\infty}(q)$,

(b) Solve the (generally overdetermined) linear system

$$
R^{\infty}=M G
$$

where $R^{\infty}=\left(R_{A_{1}^{I}}(q), R_{A_{2}^{I}}(-q), \ldots\right)^{t}$ contains both different operators $\mathscr{O}$ and different momenta $q$ giving the same $q^{2}, M$ contains the kinematical prefactors, and $G=$ $\left(G_{A}\left(q^{2}\right), G_{P}\left(q^{2}\right)\right)^{t}$.

This method has the advantage that the ratios have a well-known asymptotic behaviour, and that we only need to assume ground-state dominance in the asymptotic regime. The disadvantage is that there is no visual guidance for the goodness of the fit.

\section{Strategy II:}

(a) Define effective form factors $G_{X}^{\text {eff }}\left(q^{2}, t, t_{s}\right)$ by solving the (generally overdetermined) linear system

$$
R=M G^{\mathrm{eff}}
$$

at each $t, t_{s}$,

(b) Extract asymptotic behaviour of $G_{X}^{\mathrm{eff}}\left(q^{2}, t, t_{s}\right) \rightarrow G_{X}\left(q^{2}\right)$.

The advantage of this method is that we have some visual guidance for the goodness of the fit to $G_{X}^{\text {eff }}$, while the disadvantage is that the form factor decomposition is motivated by ground-state saturation, which will not be a good assumption at short time separations.

\subsection{Asymptotic behaviour}

With each of the two strategies, we use two different methods to extract the asymptotic behaviour:

1. The summation method [7], where we use a linear fit in $t_{s}$ to extract $R^{\infty}(q)$ from the slope of

$$
S\left(q, t_{s}\right) \equiv \sum_{t=1}^{t_{s}-1} R\left(q, t, t_{s}\right)=C+\left(R^{\infty}(q)+\mathrm{O}\left(\mathrm{e}^{-\Delta t_{s}}\right)\right) t_{s} .
$$


While this method has the advantage of not relying on specific assumptions about the excitedstate contaminations (the excited-state effects in the summed ratio are suppressed because $\Delta t_{s}>\Delta t$ by construction), it suffers from increased statistical noise. Moreover, any residual excited-state contamination may be hard to discern.

2. Explicit two-state fits of the form

$$
R\left(q, t, t_{s}\right)=R^{\infty}(q)+C_{1} \mathrm{e}^{-\Delta t}+C_{2} \mathrm{e}^{-\Delta^{\prime}\left(t_{s}-t\right)},
$$

where for our kinematics $\Delta=m_{\pi}, \Delta^{\prime}=2 m_{\pi}$ (except for $q=0$, where $\Delta=\Delta^{\prime}=2 m_{\pi}$ ). Under the assumption that the leading time dependence has been correctly identified, this method may work even for relatively short $t, t_{s}$. Its disadvantages are the model dependence inherent in the assumption that a single excited state dominates, and the need to either fix the gaps $\Delta$, $\Delta^{\prime}$ by hand, or else to perform a less stable non-linear fit.

\subsection{Momentum dependence}

From the PCAC and Goldberger-Treiman relations, we may parameterize the momentum dependence of $G_{P}$ under the assumption of pion-pole dominance as [8]

$$
G_{P}\left(Q^{2}\right)=G_{A}\left(Q^{2}\right) \frac{4 m_{N}^{2}}{Q^{2}+m_{\pi}^{2}} .
$$

We parameterize $G_{A}$ as a dipole,

$$
G_{A}\left(Q^{2}\right)=\frac{g_{A}}{\left(1+Q^{2} / M_{A}^{2}\right)^{2}},
$$

and perform a joint fit to both form factors. A Chiral Perturbation Theory-inspired parameterization and a parameterization based on the $z$-expansion [9] are under consideration.

\section{Preliminary results}

Here, we present preliminary results for the $\mathrm{N} 6\left(a \approx 0.05 \mathrm{fm}, m_{\pi} \approx 332 \mathrm{MeV}\right)$ ensemble as a representative case with comparably high statistics. We found that the signal in the $A_{0}$ channel was too noisy to be useful, and hence have omitted that channel from our analysis.

In figure 2, we compare the results obtained when using the remaining $\left(P, A_{k}\right)$ channels with those obtained using only the $A_{k}$ channels. We find that for the axial form factor $G_{A}$, neither the selection of the channels, nor the extraction strategy and excited-state suppression method used affect the result in any significant way. For the induced pseudoscalar form factor $G_{P}$, on the other hand, we find drastically different effective form factors $G_{P}^{\text {eff }}\left(Q^{2}, t, t_{s}\right)$ in strategy II, depending on whether we include or exclude the pseudoscalar operator $P$ in our basis of channels; under strategy I, this is mirrored in significantly different results obtained in the summation method when including or excluding $P$. Explicit two-state fits in strategy I give a result which is much more stable against inclusion or exclusion of the $P$ operator, and which also agrees much better with the fairly stable plateaux seen in strategy II when including $P$. On the other hand, including $P$ leads to extremely bad $\chi^{2}$ values in the least-squares solution of eq. (2.2), which appears to be driven 
mostly by the very high statistical precision of the ratios $R_{P}$, and which decrease rapidly as the time separations $t, t_{s}$ increase, indicating that a lack of ground-state dominance is the cause of the large $\chi^{2}$ values observed.

In figure 3, we compare the momentum dependence of the form factors as obtained using strategy I with either of our excited-state suppression techniques and either including or excluding $P$ among our basis of operators. We find that for the two-state fits, the inclusion or exclusion of $P$ does not affect the results for either form factor in any significant way, whereas in the case of the summation method, results for the induced pseudoscalar form factor $G_{P}$ changes by several standard deviation depending on whether $P$ is included or excluded. The summation method result including $P$ agrees well with the results from the two-state fits and yields a better description of $G_{A}$ from the combined fit (2.3-2.4), but gives much poorer $\chi^{2}$ values for the least-squares solution of eq. (2.1), than the corresponding result excluding $P$.

Our results indicate that an efficient suppression of excited-state effects is crucial also for the determination of axial form factors. In particular for the induced pseudoscalar form factor $G_{P}$, excited-state effects dominate the uncertainty of the lattice determination. The precise manner in which the axial form factors are extracted affects the amount of excited-state contamination: excited-state contributions differ significantly between different channels, making a prudent choice of operator basis crucial. Explicit two-state fits appear to be better able to extract consistent results accross channels than the summation method; this is contrary to what was found for the case of the vector form factors [3].

More details are to be contained in a forthcoming publication [10].

Acknowledgments: Our calculations were performed on the "Wilson" HPC Cluster at the Institute for Nuclear Physics, University of Mainz. We are grateful to Christian Seiwerth for technical support. We are grateful for computer time allocated to project HMZ21 on the BG/Q "JUQUEEN" computer at NIC, Jülich. This work was supported by DFG through SFB 443 and SFB 1044. TR was supported by DFG grant HA4470/3-1 and through the DFG program SFB/TRR 55. We are grateful to our colleagues within the CLS initiative for sharing ensembles.

\section{References}

[1] K. A. Olive et al. (Particle Data Group), Chin. Phys. C 38 (2014) 090001.

[2] S. Capitani et al., Phys. Rev. D 86 (2012) 074502 [arXiv:1205.0180 [hep-lat]].

[3] S. Capitani et al., Phys. Rev. D 92 (2015) 054511 [arXiv:1504.04628 [hep-lat]].

[4] S. Güsken et al., Phys. Lett. B 227 (1989) 266.

[5] M. Albanese et al. (APE Collaboration), Phys. Lett. B 192 (1987) 163.

[6] G. Martinelli and C. T. Sachrajda, Nucl. Phys. B 316 (1989) 355.

[7] L. Maiani et al., Nucl. Phys. B 293 (1987) 420; B. B. Brandt et al., Eur. Phys. J. ST 198 (2011) 79 [arXiv:1106.1554 [hep-lat]]; J. Bulava et al., JHEP 1201 (2012) 140 [arXiv:1108.3774 [hep-lat]]. J. R. Green et al., Phys. Lett. B 734 (2014) 290 [arXiv:1209.1687 [hep-lat]].

[8] G. Eichmann and C. S. Fischer, Eur. Phys. J. A 48 (2012) 9 [arXiv:1111.2614 [hep-ph]].

[9] R. J. Hill and G. Paz, Phys. Rev. D 82 (2010) 113005 [arXiv:1008.4619 [hep-ph]]; B. Bhattacharya et al., Phys. Rev. D 84 (2011) 073006 [arXiv:1108.0423 [hep-ph]].

[10] D. Djukanovic et al., in preparation. 

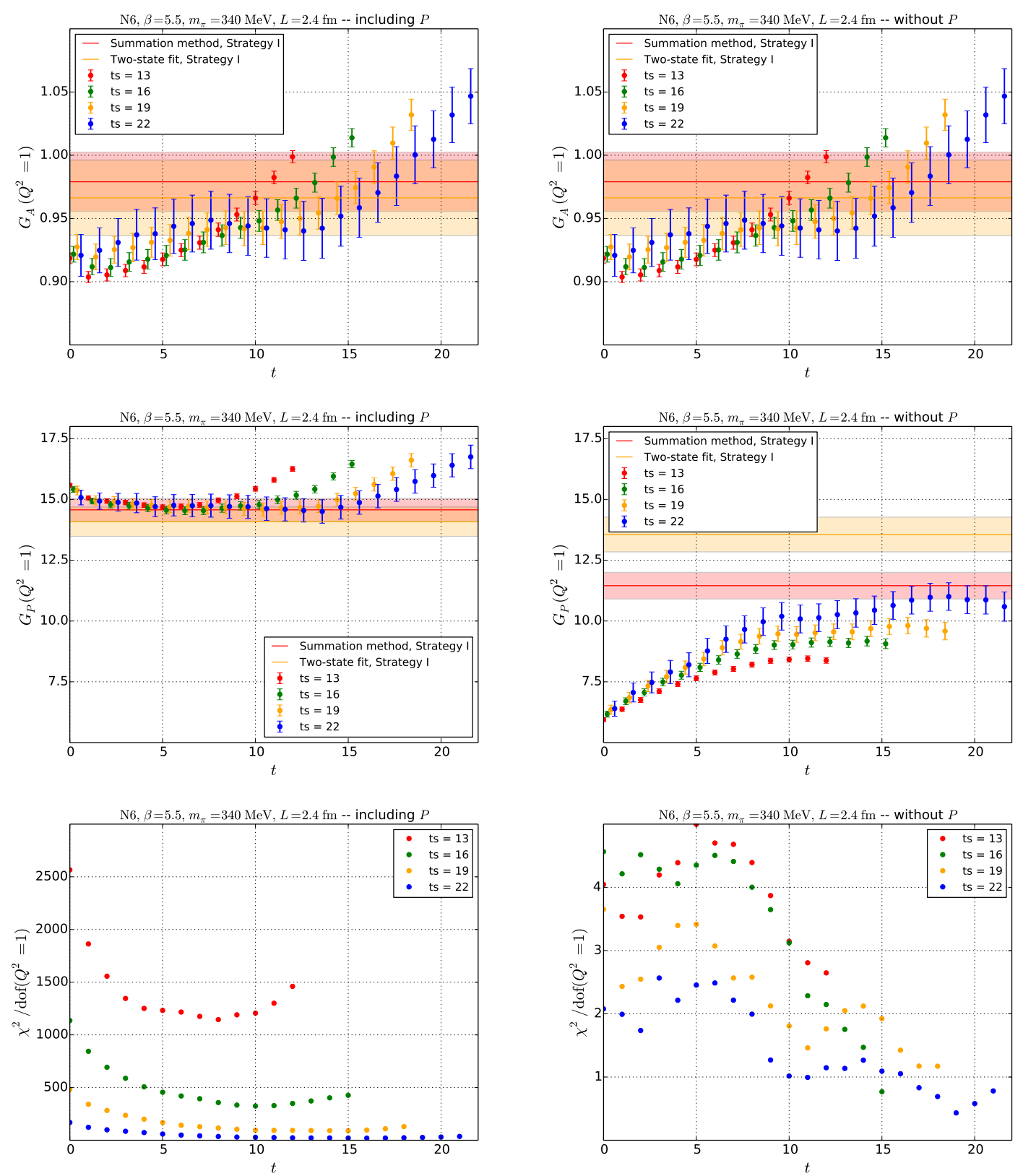

Figure 2: A comparison of the different strategies and excited-state suppression methods for $G_{A}$ (top row) and $G_{P}$ (middle row), together with the $\chi^{2}$ values (bottom row) of the least-squares solution of (2.2). Results including $P$ are shown in the left column, and results excluding $P$ in the right; in the upper two rows, the horizontal lines indicate the results obtained using strategy I, whereas the data points show the effective form factors of strategy II; note the different scales on the ordinate axes in the last row. All results are preliminary. 

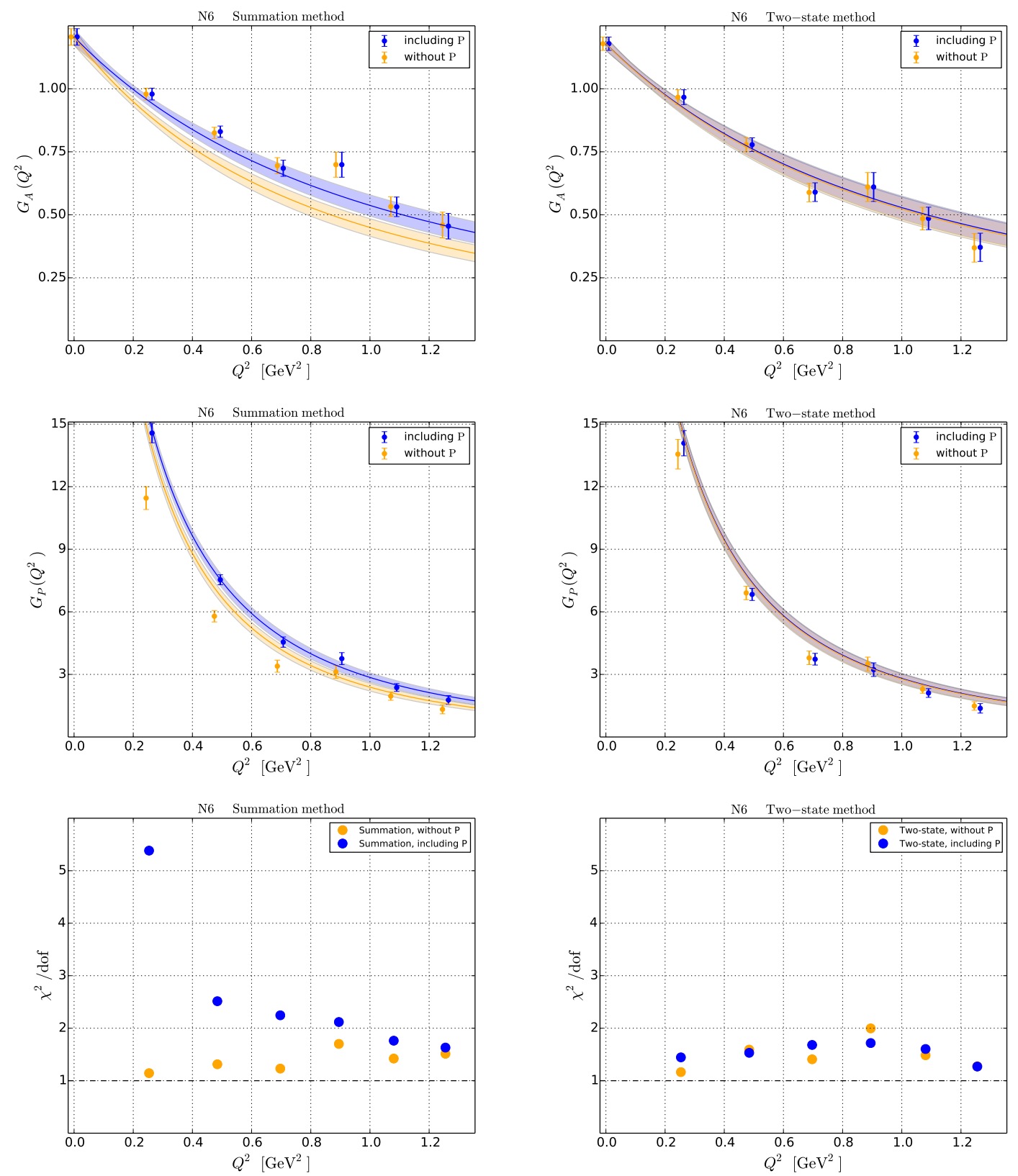

Figure 3: A comparison of results for $G_{A}$ (top row) and $G_{P}$ (middle row), together with the $\chi^{2}$ value (bottom row) of the least-squares solution of (2.1), as obtained using strategy I with different excited-state suppression methods when including (blue) or excluding (yellow) the pseudoscalar $P$ among the basis of operators. Results from the summation method are shown in the left column, whereas results using explicit two-state fits are shown in the right column. All results are preliminary. 